\title{
CONVERGENCE OF APPROXIMATE SOLUTIONS OF A DIFFERENTIAL EQUATION
}

\author{
JAN KRZYSZTOF KOWALSKI
}

Institute of Mathematics, Polish Academy of Sciences

P.O. Box 137, Śniadeckich 8, 00-950 Warszawa, Poland

E-mail: JKKOW@IMPAN.IMPAN.GOV.PL

1. Introduction and notation. The paper presents a finite-difference method for solving the differential problem

$$
\begin{gathered}
L u(t, x)=\frac{\partial u}{\partial t}(t, x)+\frac{\partial(a u)}{\partial x}(t, x)=0, \quad(t, x) \in \Omega=(0, T) \times \mathbb{R}, \\
u(0, x)=g(x), \quad x \in \mathbb{R},
\end{gathered}
$$

where $a$ is continuous and $g$ is a bounded and measurable function.

The solution of problem (1.1)-(1.2) is investigated in Section 2 (Theorem 1). Section 3 contains the definition of the finite-difference problem, in Section 4 the problem approximating (1.1)-(1.2) is formulated, and the theorems concerning the convergence of the numerical solution are stated (Theorem 2 for $g \in W_{1}^{2}(\mathbb{R})$, Theorem 3 for $\left.g \in L_{1}(\mathbb{R})\right)$. Section 5 contains some results of numerical computation. In the next sections all the results are proved.

Let us now define some function spaces which will be used in the paper. First, the spaces $L_{p}$ are defined in the usual way, and we use the following norms and moduli of continuity: if $f \in L_{p}(A), A \subset \mathbb{R}$, then

$$
\begin{aligned}
& \|f\|_{p}=\left(\int_{A}|f(x)|^{p} d x\right)^{1 / p}, \quad \omega_{p}^{k}(h, f)=\sup \left\{\left\|\Delta_{z}^{k} f\right\|_{p}: 0<z \leq h\right\}, \quad \text { where } \\
& \Delta_{z}^{k} f=\sum_{j=0}^{k}(-1)^{k-j}\left(\begin{array}{c}
k \\
j
\end{array}\right) f(\cdot+j z) \in L_{p}\left(A_{k z}\right), \quad A_{\varepsilon}=\{x \in A:(x, x+\varepsilon) \subset A\} .
\end{aligned}
$$

Next, let us consider the two-dimensional case. Let $I=[0, T]$, and let $\varrho_{1}$ :

1991 Mathematics Subject Classification: 65M06, 65M15, 41A15.

The paper is in final form and no version of it will be published elsewhere. 
$I \rightarrow \mathbb{R} \cup\{-\infty\}, \varrho_{2}: I \rightarrow \mathbb{R} \cup\{+\infty\}$ be continuous functions, $Q=\{(t, x):$ $\left.t \in I, \varrho_{1}(t)<x<\varrho_{2}(t)\right\}, Q^{t}=\{x:(t, x) \in Q\}$.

The space $\mathcal{L}_{C}(Q)$ is defined as the set of all functions $u$ which are measurable on $Q$ and such that for each $t \in I, u(t, \cdot) \in L_{1}\left(Q^{t}\right)$ and

$$
\begin{aligned}
& \|u\|_{*}=\sup \left\{\left\|u^{t}\right\|_{1}: t \in I\right\}<\infty, \quad\left\|\mathcal{P} u^{s}-\mathcal{P} u^{t}\right\|_{1} \rightarrow 0 \quad \text { as } s \rightarrow t \\
& \left(u^{t}=u(t, \cdot)\right)
\end{aligned}
$$

(the function $\mathcal{P} u^{s}$ is defined on $\mathbb{R}$ by $\mathcal{P} u^{s}(x)=u^{s}(x)$ if $x \in Q^{s}, \mathcal{P} u^{s}(x)=0$ if $\left.x \in \mathbb{R} \backslash Q^{s}\right) ;\|\cdot\|_{*}$ is the norm in $\mathcal{L}_{C}(Q)$. The following moduli of continuity will be used in $\mathcal{L}_{C}(Q)$ :

$$
\begin{aligned}
& \omega_{*}^{k}(h, u)=\sup \left\{\omega_{1}^{k}\left(h, u^{t}\right): t \in I\right\}, \\
& \omega_{0}(h, u)=\sup \left\{\left\|\mathcal{P} u^{t}-\mathcal{P} u^{s}\right\|_{1}: s, t \in I,|s-t| \leq h\right\} .
\end{aligned}
$$

It can be proved (see Section 7 ) that

$$
\text { (1.4) if } u \in \mathcal{L}_{C}(Q) \text { then } \omega_{*}^{k}(h, u) \rightarrow 0 \text { as } h \rightarrow 0 \text {; }
$$

the fact that $\omega_{0}(h, u) \rightarrow 0$ as $h \rightarrow 0$ directly follows from the definition of $\mathcal{L}_{C}(Q)$.

It will be convenient to introduce $C_{\text {mon }}$, the set of all nondecreasing functions $\sigma$ such that $\lim _{h \rightarrow 0} \sigma(h)=0$. Formula (1.4) can thus be written as $\omega_{*}^{k}(\cdot, u) \in C_{\text {mon }}$.

Finally, since we are interested mostly in the derivatives with respect to $x$, we use the notation

$$
D^{k} u=D_{x}^{k} u=\frac{\partial^{k}}{\partial x^{k}} u .
$$

2. Solution of the differential problem. To consider the properties of the solution of problem (1.1)-(1.2) we use the characteristics of the operator $L$, that is, continuous functions $\varphi: I \rightarrow \mathbb{R}$ satisfying

$$
\frac{d}{d t} \varphi(t)=a(t, \varphi(t)), \quad t \in I .
$$

Throughout this paper we assume that

$$
a \in C(\Omega), \quad D a \in \mathcal{L}_{C}(\Omega) \cap L_{\infty}(\Omega),
$$

and we use the notation

$$
A=\|a\|_{\infty}, \quad A^{\prime}=\|D a\|_{\infty}, \quad A_{*}^{\prime}=\|D a\|_{*} .
$$

With this assumption it can be proved that if $\varphi, \psi$ are two characteristics then $e^{A^{\prime}(s-t)}|\varphi(t)-\psi(t)| \leq|\varphi(s)-\psi(s)| \leq e^{A^{\prime}(t-s)}|\varphi(t)-\psi(t)| \quad$ if $0 \leq s<t \leq T$.

Hence, no two characteristics have common points and for each $(t, x) \in \Omega$ we can define the function $\lambda(\cdot, t, x)$ as the characteristic passing through $(t, x)$, that is,

$$
\frac{\partial}{\partial s} \lambda(s, t, x)=a(s, \lambda(s, t, x)), \quad \lambda(t, t, x)=x, \quad \text { if } 0 \leq s<t \leq T ;
$$

we also use the function $\kappa$ defined by

$$
\kappa(s, t, x)=(x-\lambda(s, t, x)) /(t-s) \quad(0 \leq s<t \leq T, x \in \mathbb{R}) .
$$


We thus have

$$
\begin{aligned}
& \lambda(s, t, x)=x-\int_{s}^{t} a(\theta, \lambda(\theta, t, x)) d \theta, \\
& \kappa(s, t, x)=\frac{1}{t-s} \int_{s}^{t} a(\theta, \lambda(\theta, t, x)) d \theta ;
\end{aligned}
$$

differentiating (2.3) we get

$$
\begin{aligned}
& D \lambda(s, t, x)=\exp \left(-\int_{s}^{t} D a(\theta, \lambda(\theta, t, x)) d \theta\right) \\
& D \kappa(s, t, x)=\frac{1}{t-s} \int_{s}^{t} D a(\theta, \lambda(\theta, t, x)) D \lambda(\theta, t, x) d \theta .
\end{aligned}
$$

Therefore,

$$
\Lambda_{s t}^{-1} \leq D \lambda(s, t, x) \leq \Lambda_{s t}=e^{A^{\prime}(t-s)} \quad \text { if } 0 \leq s<t \leq T, x \in \mathbb{R} .
$$

It also follows from (2.3) and (2.4) that

$$
\|\kappa\|_{\infty} \leq A, \quad\|D \kappa(s, t, \cdot)\|_{1} \leq A_{*}^{\prime} .
$$

If $u$ is a solution of (1.1)-(1.2), then

$$
u(t, x)=D \lambda(0, t, x) g(\lambda(0, t, x)),
$$

where, as in (1.5), $D^{k} \lambda=\partial^{k} \lambda / \partial x^{k}$. This formula allows us to investigate the properties of the solution of (1.1)-(1.2). First, we see that

$$
\left\|u^{t}\right\|_{1}=\left\|u^{0}\right\|_{1} \quad \text { for each } t \in I .
$$

Next, the following theorem is true.

Theorem 1. Let $\varrho \in C^{1}(I), \Omega_{<}=\{(t, x): t \in I, x<\varrho(t)\}, \Omega_{>}=\{(t, x):$ $t \in I, x>\varrho(t)\}, a_{<}=\left.a\right|_{\Omega_{<}}, a_{>}=\left.a\right|_{\Omega_{>}}$. We assume that (2.1) is satisfied and

$$
\begin{gathered}
D^{2} a_{<} \in \mathcal{L}_{C}\left(\Omega_{<}\right) \cap L_{\infty}\left(\Omega_{<}\right), \quad D^{2} a_{>} \in \mathcal{L}_{C}\left(\Omega_{>}\right) \cap L_{\infty}\left(\Omega_{>}\right), \\
\exists \beta_{0}>0 \forall s \in I\left|a(s, \varrho(s))-\varrho^{\prime}(s)\right| \geq \beta_{0},
\end{gathered}
$$

and we use notation (2.2) and

(2.11) $\quad\left\|\varrho^{\prime}\right\|_{\infty}=M, \quad \omega_{\infty}^{1}\left(\varepsilon, \varrho^{\prime}\right)=\sigma_{0}(\varepsilon), \quad \omega_{0}(h, D a)=\sigma_{10}(h)$,

(2.12) $\left\|a_{\varrho \Delta}^{\prime}\right\|_{\infty}=A_{\Delta}^{\prime}$, where $a_{\varrho \Delta}^{\prime}=a_{\varrho+}^{\prime}-a_{\varrho-}^{\prime}, a_{\varrho \pm}^{\prime}=D a(\cdot, \varrho(\cdot) \pm 0)$,

(2.13) $\max \left(\left\|D^{2} a_{<}\right\|_{\infty},\left\|D^{2} a_{>}\right\|_{\infty}\right)=A^{\prime \prime}, \quad\left\|D^{2} a_{<}\right\|_{*}+\left\|D^{2} a_{>}\right\|_{*}=A_{*}^{\prime \prime}$,

$$
\begin{aligned}
\omega_{0}\left(h, D^{2} a_{<}\right)+\omega_{0}\left(h, D^{2} a_{>}\right) & =\sigma_{20}(h), \\
\max \left(\omega_{*}^{1}\left(h, D^{2} a_{<}\right), \omega_{*}^{1}\left(h, D^{2} a_{>}\right)\right) & =\sigma_{2}(h) .
\end{aligned}
$$


Then for every $s, t, D^{2} \lambda(s, t, \cdot) \in L_{1}(\mathbb{R}) \cap L_{\infty}(\mathbb{R})$ and

$$
\begin{gathered}
\left\|D^{2} \lambda(s, t, \cdot)\right\|_{\infty} \leq e^{2 A^{\prime}(t-s)}\left((t-s) A^{\prime \prime}+A_{\Delta}^{\prime} / \beta_{0}\right), \\
\left\|D^{2} \lambda(s, t, \cdot)\right\|_{1} \leq e^{A^{\prime}(t-s)}(t-s)\left(A_{*}^{\prime \prime}+A_{\Delta}^{\prime}\right),
\end{gathered}
$$

$$
\begin{aligned}
& \text { there exists a function } \sigma \in C_{\mathrm{mon}} \text { such that } \\
& \qquad \omega_{1}^{1}\left(h, D^{2} \lambda(s, t, \cdot)\right) \leq \sigma(h) \quad \text { if } 0 \leq s<t \leq T .
\end{aligned}
$$

In the further considerations we use the operators

$$
E(s, t): L_{\infty}(\mathbb{R}) \rightarrow L_{\infty}(\mathbb{R}) \quad(0 \leq s<t \leq T)
$$

defined by the formula

$$
[E(s, t) f](x)=D \lambda(s, t, x) f(\lambda(s, t, x)) \quad \forall x \in \mathbb{R} .
$$

It follows from (2.7) that the solution of (1.1)-(1.2) satisfies

$$
u^{t}=E(s, t) u^{s} \quad \text { if } 0 \leq s<t \leq T .
$$

3. Finite-difference problem. In order to define an approximate solution, we introduce the mesh

$$
\begin{gathered}
\Omega_{h}=\left\{(t, x) \in \Omega: t=n \tau, x=m h, m, n \in \mathbb{Z}, 0 \leq n \leq N_{h}\right\}, \quad N_{h}=[T / \tau], \\
\Omega_{h}^{\prime}=\left\{(n \tau, m h) \in \Omega_{h}: n \leq N_{h}-1\right\}, \quad \mathbb{R}_{h}=\{x \in \mathbb{R}: x=m h, m \in \mathbb{Z}\},
\end{gathered}
$$

where $h$ (the step size) is a parameter from the interval $(0,1), \tau=\mu h$, and $\mu$ is a fixed number (independent of $h$ ).

Let $m(A)$ be the set of all functions defined on $A$. We introduce the following notation for any $w_{h} \in m\left(\mathbb{R}_{h}\right)$ and $v_{h} \in m\left(\Omega_{h}\right)$ :

$$
\begin{gathered}
v_{m}^{n}=v_{h}(n \tau, m h), \quad v^{n}=v_{h}(n \tau, \cdot), \quad w_{m}=w_{h}(m h), \\
\left\|w_{h}\right\|_{\infty}=\sup \left\{\left|w_{m}\right|: m \in \mathbb{Z}\right\}, \quad\left\|w_{h}\right\|_{1}=h \sum_{m \in \mathbb{Z}}\left|w_{m}\right|, \\
\left\|v_{h}\right\|_{\infty}=\max \left\{\left\|v^{n}\right\|_{\infty}: 0 \leq n \leq N_{h}\right\}, \\
\left\|v_{h}\right\|_{*}=\max \left\{\left\|v^{n}\right\|_{1}: 0 \leq n \leq N_{h}\right\} .
\end{gathered}
$$

Next, we introduce the difference operator, $L_{h}: m\left(\Omega_{h}\right) \rightarrow m\left(\Omega_{h}^{\prime}\right)$, by

$$
\left(L_{h} v_{h}\right)_{m}^{n}=\frac{1}{\tau}\left[v_{m}^{n+1}-\frac{1}{2}\left(v_{m+1}^{n}+v_{m-1}^{n}\right)\right]+\frac{1}{2 h}\left[\alpha_{m+1}^{n} v_{m+1}^{n}-\alpha_{m-1}^{n} v_{m-1}^{n}\right],
$$

for $v_{h} \in m\left(\Omega_{h}\right), m \in \mathbb{Z}, n=0,1, \ldots, N_{h}-1$,

where $\alpha \in m\left(\Omega_{h}^{\prime}\right)$ is given, and we formulate the following difference problem: find $v_{h} \in m\left(\Omega_{h}\right)$ such that

$$
\left(L_{h} v_{h}\right)_{m}^{n}=0 \quad \text { for }(n \tau, m h) \in \Omega_{h}^{\prime}, \quad v^{0} \in m\left(\mathbb{R}_{h}\right) \text { given. }
$$

It can be easily seen that problem (3.3) has a unique solution $v_{h}$, and

$$
\text { if } \mu\|\alpha\|_{\infty} \leq 1 \text { then }\left\|v_{h}\right\|_{*} \leq\left\|v^{0}\right\|_{1} \text {. }
$$


4. Approximation of the differential equation. Let us define the operators of restriction (see [1]), $r_{h}^{0}: L_{1}^{\text {loc }}(\mathbb{R}) \rightarrow m\left(\mathbb{R}_{h}\right), r_{h}: \mathcal{L}_{C}(\Omega) \rightarrow m\left(\Omega_{h}\right)$, by the formulas

$$
\begin{aligned}
& \left(r_{h}^{0} f\right)_{m}=\frac{1}{2 h} \int_{(m-1) h}^{(m+1) h} f(x) d x \quad \text { if } f \in L_{\infty}(\mathbb{R}), \\
& \left(r_{h} u\right)_{m}^{n}=\left(r_{h}^{0} u^{n \tau}\right)_{m} \quad \text { if } u \in \mathcal{L}_{C}(\Omega)
\end{aligned}
$$

$\left(u^{t}\right.$ is defined in (1.3)).

We consider problem (3.3) where

$$
\alpha_{m}^{n}=a(n \tau, m h), \quad v^{0}=r_{h}^{0} g,
$$

and assume that

$$
\mu A=\mu\|a\|_{\infty} \leq 1 .
$$

If the operator $F_{h}: L_{\infty}(\mathbb{R}) \rightarrow m\left(\Omega_{h}\right)$ is defined by the formula

$$
\left(F_{h} g\right)^{0}=r_{h}^{0} g,
$$

$$
\begin{array}{r}
\left(F_{h} g\right)_{m}^{n+1}=\frac{1}{2}\left(1-\mu \alpha_{m+1}^{n}\right)\left(F_{h} g\right)_{m+1}^{n}+\frac{1}{2}\left(1+\mu \alpha_{m-1}^{n}\right)\left(F_{h} g\right)_{m-1}^{n}, \\
(n \tau, m h) \in \Omega_{h}^{\prime},
\end{array}
$$

then $F_{h} g$ is the unique solution of problem (3.3), (4.2).

Let $u$ be the solution of problem (1.1)-(1.2) and $v_{h}$ the solution of (3.3), (4.2). Our purpose is to estimate the error of approximation, that is, the function

$$
z_{h}=v_{h}-r_{h} u
$$

in the norm $\|\cdot\|_{*}$.

Using definitions (2.17) and (4.4), we can write the error $z_{h}$ in the form

$$
z_{h}=F_{h} g-r_{h}(E(0, \cdot) g)
$$

that is, $z^{n}=\left(F_{h} g\right)^{n}-r_{h}^{0}(E(0, n \tau) g)$ for $0 \leq n \leq N_{h}$.

The estimate for $\left\|z_{h}\right\|_{*}$ depends on the regularity of the solution $u$, and hence of $g$ and $a$. First, we have the following result.

Theorem 2. Assume that conditions (2.1), (4.3) are satisfied and that $D^{2} \lambda$ satisfies (2.16), and use notation (2.2), (2.11) and

$$
\|D \lambda\|_{\infty}=\Lambda, \quad\left\|D^{2} \lambda(0, \cdot, \cdot)\right\|_{*}=\Lambda^{\prime}, \quad \omega_{*}^{1}(h, D a)=\sigma_{1}(h) .
$$

Then for each $g \in W_{1}^{2}(\mathbb{R})$,

$$
\left\|z_{h}\right\|_{*} \leq M_{1}(h)\|D g\|_{1}+M_{2} h\left\|D^{2} g\right\|_{1},
$$

where $M_{1}(h)=T\left\{\left(\Lambda+\Lambda^{\prime} / 4\right) \sigma_{10}(\mu h)+\frac{5}{2} \Lambda \sigma_{1}(h)+\left(\mu_{1} / 2\right) \sigma(h)+h A^{\prime}\left(e^{A^{\prime} \mu h}(\Lambda(1+\right.\right.$ $\left.\left.\left.\left.(\mu / 2) A_{*}^{\prime}\right)+\Lambda^{\prime} / 2\right)+\Lambda / 2+\Lambda^{\prime} / 4\right)\right\}, M_{2}=T \Lambda \mu_{1}\left(\Lambda+\frac{3}{2} \Lambda^{\prime}\right), \mu_{1}=81 /(4 \mu)$. 
In the next theorem the initial function $g$ has a lower regularity.

THEOREM 3. Let the assumptions of Theorem 2 be satisfied. If $g \in L_{1}(\mathbb{R}) \cap$ $L_{\infty}(\mathbb{R})$ then

$$
\left\|z_{h}\right\|_{*} \leq M_{3}(h) \omega_{1}^{1}(\psi(h), g)+M_{4} \omega_{1}^{2}(\psi(h), g),
$$

where $\psi(h)=\max \left(\sqrt{h}, M_{1}(h)\right), M_{3}(h)=M_{1}(h) / \psi(h), M_{4}=\frac{13}{3}+3 M_{2}$, and $M_{1}(h), M_{2}$ are taken from Theorem 2 .

These theorems are proved in Section 8.

5. Numerical examples. We present here some numerical results. We consider problem (1.1)-(1.2) where $a$ is constant, and $g$ has two values:

$$
g(x)=u_{-} \quad \text { if } x<0, \quad g(x)=u_{+} \quad \text { if } x>0 .
$$

In this case

$$
u(t, x)=u_{-} \quad \text { if } x<a t, \quad u(t, x)=u_{+} \quad \text { if } x>a t .
$$

We also consider problem (3.3) with the coefficients given by (4.2), and the error $z_{h}$ defined by (4.5). The norm of $z_{h}$ can be estimated with the use of Theorem 3 , where $\psi(h)=\sqrt{h}, M_{3}(h)=0, M_{4}=13 / 3+243 /(4 \mu)$. We also see that $\omega_{1}^{1}(\varepsilon, g)=$ $\varepsilon\left|u_{+}-u_{-}\right|, \omega_{1}^{2}(\varepsilon, g)=2 \varepsilon\left|u_{+}-u_{-}\right|$. Theorem 3 says that

$$
\left\|z_{h}\right\|_{*} \leq 2 M_{4}\left|u_{+}-u_{-}\right| \sqrt{h}
$$

Below, we present some results of computation for $T=1$ and different values of $a, u_{-}, u_{+}, h$.

\begin{tabular}{|c|c|c|}
\hline \multicolumn{3}{|c|}{$u_{-}=1.00, u_{+}=2.00, a=0.00, \mu=1.00$} \\
\hline$h$ & $\left\|z^{N}\right\|_{1}$ & $\left\|z^{N}\right\|_{1} / \sqrt{h}$ \\
\hline 0.010000000 & 0.051630 & 0.516302 \\
0.005000000 & 0.037469 & 0.529891 \\
0.002500000 & 0.026986 & 0.539718 \\
0.001250000 & 0.019331 & 0.546776 \\
0.000625000 & 0.013796 & 0.551822 \\
0.000312500 & 0.009818 & 0.555417 \\
\hline
\end{tabular}

\begin{tabular}{|c|c|c|}
\hline \multicolumn{3}{|c|}{$u_{-}=0.00, u_{+}=1.00, a=1.60, \mu=0.50$} \\
\hline$h$ & $\left\|z^{N}\right\|_{1}$ & $\left\|z^{N}\right\|_{1} / \sqrt{h}$ \\
\hline .025000000 & 0.064448 & 0.407604 \\
.012500000 & 0.047719 & 0.426813 \\
.006250000 & 0.034880 & 0.441199 \\
.003125000 & 0.025255 & 0.451779 \\
.001562500 & 0.018162 & 0.459467 \\
.000781250 & 0.012997 & 0.465005 \\
\hline
\end{tabular}

\begin{tabular}{|c|c|c|}
\hline \multicolumn{3}{|c|}{$u_{-}=2.00, u_{+}=3.00, a=0.80, \mu=1.00$} \\
\hline$h$ & $\left\|z^{N}\right\|_{1}$ & $\left\|z^{N}\right\|_{1} / \sqrt{h}$ \\
\hline 0.020000000 & 0.039135 & 0.276724 \\
0.010000000 & 0.029301 & 0.293008 \\
0.005000000 & 0.021596 & 0.305414 \\
0.002500000 & 0.015732 & 0.314644 \\
0.001250000 & 0.011363 & 0.321402 \\
0.000625000 & 0.008157 & 0.326297 \\
\hline
\end{tabular}

\begin{tabular}{|c|c|c|}
\hline \multicolumn{3}{|c|}{$u_{-}=2.00, u_{+}=3.00, a=0.80, \mu=0.50$} \\
\hline$h$ & $\left\|z^{N}\right\|_{1}$ & $\left\|z^{N}\right\|_{1} / \sqrt{h}$ \\
\hline 0.020000000 & 0.093952 & 0.664340 \\
0.010000000 & 0.068316 & 0.683164 \\
0.005000000 & 0.049276 & 0.696864 \\
0.002500000 & 0.035337 & 0.706746 \\
0.001250000 & 0.025238 & 0.713832 \\
0.000625000 & 0.017972 & 0.718892 \\
\hline
\end{tabular}




\begin{tabular}{|c|c|c|}
\hline \multicolumn{3}{|c|}{$u_{-}=0.80, u_{+}=2.10, a=-1.00, \mu=0.60$} \\
\hline$h$ & $\left\|z^{N}\right\|_{1}$ & $\left\|z^{N}\right\|_{1} / \sqrt{h}$ \\
\hline 0.020833333 & 0.096772 & 0.670454 \\
0.010416667 & 0.070888 & 0.694555 \\
0.005208333 & 0.051405 & 0.712290 \\
0.002604167 & 0.037007 & 0.725180 \\
0.001302083 & 0.026503 & 0.734471 \\
0.000651042 & 0.018910 & 0.741128 \\
\hline
\end{tabular}

\begin{tabular}{|c|c|c|}
\hline \multicolumn{3}{|c|}{$u_{-}=-1.00, u_{+}=1.00, a=0.17, \mu=0.80$} \\
\hline$h$ & $\left\|z^{N}\right\|_{1}$ & $\left\|z^{N}\right\|_{1} / \sqrt{h}$ \\
\hline 0.016666667 & 0.145127 & 1.124150 \\
0.008333333 & 0.105862 & 1.159659 \\
0.004166667 & 0.076573 & 1.186262 \\
0.002083333 & 0.054977 & 1.204496 \\
0.001041667 & 0.039304 & 1.217783 \\
0.000520833 & 0.028004 & 1.227092 \\
\hline
\end{tabular}

Thus, we observe that the convergence of $\left\|z_{h}\right\|_{*}$ is of order $\sqrt{h}$, as stated in Theorem 3.

6. Auxiliary formulas and lemmas. All the results presented in this section are proved in Section 7 .

Let us start from a lemma which allows us to obtain estimates for functions of low regularity.

LEMma 4. Let $X$ be a Banach space and consider the operator $\Phi: L_{p}\left(\mathbb{R}^{n}\right) \rightarrow$ $X$. Assume that there exist nonnegative numbers $M, \eta, C_{0}, C_{1}, \ldots, C_{k}$ such that

$$
\begin{gathered}
\forall g, g^{\prime} \in L_{p}\left(\mathbb{R}^{n}\right) \quad\left\|\Phi(g)-\Phi\left(g^{\prime}\right)\right\|_{X} \leq M\left\|g-g^{\prime}\right\|_{p}, \\
\forall f \in W_{p}^{k}\left(\mathbb{R}^{n}\right) \quad\|\Phi(f)\|_{X} \leq \eta \sum_{l=0}^{k} C_{l}|f|_{p}^{(l)} .
\end{gathered}
$$

Then there exist constants $N, N_{0}, \ldots, N_{k}$ (depending only on $\left.k, n, p\right)$ such that for every $g \in L_{p}\left(\mathbb{R}^{n}\right)$,

$$
\|\Phi(g)\|_{X} \leq\left(M N+N_{k} C_{k}\right) \omega_{p}^{k}\left(\eta^{1 / k}, g\right)+\sum_{l=0}^{k-1} \eta^{1-l / k} N_{l} C_{l} \omega_{p}^{l}\left(\eta^{1 / k}, g\right) .
$$

It can be checked that

$$
\begin{aligned}
& \text { if } n=k=p=1, \text { then } N=\frac{3}{2}, N_{0}=N_{1}=1 ; \\
& \text { if } n=p=1, k=2, \text { then } N=\frac{13}{3}, N_{0}=N_{1}=N_{2}=3 .
\end{aligned}
$$

The next two lemmas will be used in the proof of Theorem 2 .

LEMmA 5. Let the operators $\pi_{h}: L_{1}^{\text {loc }}(\mathbb{R}) \rightarrow L_{\infty}^{\text {loc }}(\mathbb{R})\left(h \in H \subset \mathbb{R}_{+}\right)$be defined by

$$
\left(\pi_{h} f\right)(x)=\int_{\mathbb{R}} W_{h}(x, z) f(x+z h) d z,
$$

where $W_{h}$ are bounded measurable functions on $\mathbb{R}^{2}$ satisfying

$$
\forall h \in H \exists \beta_{h}>0 \forall x \in \mathbb{R} \quad \operatorname{supp} W_{h}(x, \cdot) \subset\left[-\beta_{h}, \beta_{h}\right] .
$$

For $x \in \mathbb{R}$ let $\psi_{j h}(x)=\int_{\mathbb{R}} W_{h}(x, z) z^{j} d z$. If $\psi_{j h} \in L_{p(j)}(\mathbb{R})(j=0,1, \ldots, k-1$, 
$1 \leq p(j) \leq \infty)$, then

$$
\forall f \in W_{1}^{k}(\mathbb{R}) \quad\left\|\pi_{h} f\right\|_{1} \leq \sum_{j=0}^{k} \vartheta_{j h} h^{j}\left\|D^{j} f\right\|_{p^{\prime}(j)}+\vartheta_{k h}^{\prime} h^{k} \omega_{1}^{1}\left(h \beta_{h}, D^{k} f\right),
$$

where $\vartheta_{j h}=(1 / j !)\left\|\psi_{j h}\right\|_{p(j)}, \vartheta_{k h}^{\prime}=(2 /(k+1) !) \beta_{h}^{k+1}\left\|W_{h}\right\|_{\infty}, 1 / p(j)+1 / p^{\prime}(j)=1$.

LEMMA 6. Let $f \in L_{1}(\mathbb{R})$,

$$
\varphi_{m, h}(f)=\left|\int_{(m-1) h}^{(m+1) h} f(x) d x-\int_{\phi_{m-1}}^{\phi_{m+1}} f(y) d y\right| \quad \text { for } m \in \mathbb{Z}
$$

and $\left|\phi_{m}-m h\right| \leq h, h e^{-B h} \leq \Delta \phi_{m} \leq h e^{B h}$. Then

$$
\sum_{m \in \mathbb{Z}} \varphi_{m, h}(f) \leq 10 \omega_{1}^{1}(h, f)+2 B h e^{B h}\|f\|_{1} .
$$

The following lemma is needed for proving Theorem 1.

LEMma 7. Let $f \in L_{1}(a, b), \varphi:[c, d] \rightarrow[a, b], 0<P_{2}^{-1} \leq D \varphi(x) \leq P_{1}$ for almost every $x \in[c, d]$. Then

$$
\omega_{1}^{1}(h, f(\varphi(\cdot)) D \varphi) \leq\left(3+P_{1}\right) \omega_{1}^{1}(h, \mathcal{P} f)+P_{2} \omega_{\infty}^{1}(h, D \varphi)\|f\|_{1} .
$$

Finally, we formulate some properties of measurable functions:

(6.10) if $g \in W_{1}^{1}(a, b)$ then $g \in L_{\infty}(a, b)$ and $\|g\|_{\infty} \leq(2 /(b-a))\|g\|_{1}+\|D g\|_{1}$,

(6.11) if $g \in W_{1}^{1}(\mathbb{R})$ then $g \in L_{\infty}(\mathbb{R})$ and $\|g\|_{\infty} \leq \frac{1}{2}\|D g\|_{1}$,

and a formula which can be proved with the use of the mean value theorem:

$$
\forall a, b \in \mathbb{R} \exists \xi \in(0,1) \quad e^{a}-e^{b}=\left(\xi e^{a}+(1-\xi) e^{b}\right)(a-b) .
$$

7. Proofs of auxiliary formulas. In this section all the results from the previous section and formula (1.4) are proved.

Proof of Lemma 4. We first give a definition of multivariate box splines (cf. [1] or [2]), which will be used in the proof. We introduce the class $\mathcal{S}_{k}$ of all systems of vectors from $\mathbb{Z}^{n}$ of the form

$$
Y=\left[x_{1}, \ldots, x_{r}\right], \quad \text { where } r>n k, x_{l k+j}=e_{l+1} \text { if } 0 \leq l \leq n-1,1 \leq j \leq k
$$

( $e_{i}$ is the unit vector of the $i$ th axis) which satisfy the condition: each subsystem of $Y$ consisting of $r-k$ vectors spans the space $\mathbb{R}^{n}$.

The multivariate box spline $B_{Y}$ is the function satisfying the identity

$$
\int_{\mathbb{R}^{n}} B_{Y}(x) f(x) d x=\int_{0}^{1} \ldots \int_{0}^{1} f\left(\sum_{j=1}^{r} \xi_{j} x_{j}\right) d \xi_{1} \ldots d \xi_{r}
$$

for every $f \in C\left(\mathbb{R}^{n}\right)$. 
Let $g \in L_{p}\left(\mathbb{R}^{n}\right)$ be fixed. If $f \in W_{p}^{k}\left(\mathbb{R}^{n}\right)$ is an arbitrary function, it follows from the triangle inequality and assumptions (6.1), (6.2) that

$$
\|\Phi(g)\|_{X} \leq M\|g-f\|_{p}+\eta \sum_{l=0}^{k} C_{l}|f|_{p}^{(l)}
$$

Now, as in the proof of Lemma 2 in [2], we construct $f$ for which the right-hand side of (7.1) can be estimated by the right-hand side of (6.3).

Let $Y \in \mathcal{S}_{k}$ and let $B_{Y}$ be the corresponding box spline. Let $t>0$ and

$$
f_{t}(x)=-\sum_{j=1}^{k}(-1)^{j}\left(\begin{array}{l}
k \\
j
\end{array}\right) \int_{\mathbb{R}^{n}} B_{Y}(y) g(x+j t y) d y
$$

the number $t$ will be chosen later. It is shown in [2] that

$$
\left\|g-f_{t}\right\|_{p} \leq N \omega_{p}^{k}(t, g), \quad\left|f_{t}\right|_{p}^{(l)} \leq t^{-l} N_{l} \omega_{p}^{l}(t, g), \quad l=0,1, \ldots, k,
$$

where $N$ and $N_{l}$ depend only on $k, p$ and $Y$. Taking $t=\eta^{1 / k}$ we thus obtain the estimate

$$
M\left\|g-f_{t}\right\|_{p}+\eta \sum_{l=0}^{k} C_{l}|f|_{p}^{(l)} \leq M N \omega_{p}^{k}\left(\eta^{1 / k}, g\right)+\sum_{l=0}^{k} \eta^{1-l / k} N_{l} C_{l} \omega_{p}^{l}\left(\eta^{1 / k}, g\right) .
$$

Inequality (6.3) follows from this formula and (7.1).

Pro of of (6.4). We use here the notation from the proof of Lemma 4 . Let $B_{l}$ $\left(l \in \mathbb{Z}_{+}\right)$be the Schoenberg splines satisfying the recurrence relation $B_{0}=\chi_{[0,1]}$, $B_{l+1}=\int_{-1}^{0} B_{l}(x+\xi) d \xi$. Then $B_{l}$ is the spline $B_{Y}$ where $Y=[1, \ldots, 1] \in \mathbb{Z}^{l+1}$. Defining the operator $\mathcal{M}_{\varepsilon}^{k}$ by $\mathcal{M}_{\varepsilon}^{k} g(x)=\int_{\mathbb{R}} B_{k}(y) g(x+\varepsilon y) d y$, we see that $f_{t}=$ $-\sum_{j=1}^{k}(-1)^{j}\left(\begin{array}{l}k \\ j\end{array}\right) \mathcal{M}_{j t}^{k} g$. Hence $g-f_{t}=(-1)^{k} \int_{\mathbb{R}} B_{k}(y) \Delta_{t y}^{k} g(\cdot) d y$, and consequently

$$
\left\|g-f_{t}\right\|_{1} \leq \int_{\mathbb{R}} B_{k}(y) \int_{\mathbb{R}}\left|\Delta_{t y}^{k} g(x)\right| d x d y=\int_{\mathbb{R}} B_{k}(y)\left\|\Delta_{t y}^{k} g\right\|_{1} d y .
$$

Since for $j \in \mathbb{Z},\left\|\Delta_{t j}^{k} g\right\|_{1} \leq j^{k}\left\|\Delta_{t}^{k} g\right\|_{1}$, and $\left\|\Delta_{t y}^{k} g\right\|_{1} \leq \omega_{1}^{k}(t j, g)$ if $0<y \leq j$, we obtain

$$
\left\|g-f_{t}\right\|_{1} \leq N \omega_{1}^{k}(t, g), \quad \text { where } N=\sum_{j=1}^{k+1} j^{k} \int_{j-1}^{j} B_{k}(y) d y .
$$

Hence $N=\frac{3}{2}$ if $k=1, N=\frac{13}{3}$ if $k=2$.

It is shown in the proof of Lemma 2 in [2] that (in the one-dimensional case) $D^{l} \mathcal{M}_{\varepsilon}^{k} g=\varepsilon^{-l} \mathcal{M}_{\varepsilon}^{k-l}\left(\Delta_{\varepsilon}^{l} g\right),\left\|\mathcal{M}_{\varepsilon}^{k} g\right\|_{1} \leq\|g\|_{1}$. Thus

$\left\|D^{l} f_{t}\right\|_{1} \leq \sum_{j=1}^{k}\left(\begin{array}{l}k \\ j\end{array}\right)(j t)^{-l}\left\|\mathcal{M}_{j t}^{k-l}\left(\Delta_{j t}^{l} g\right)\right\|_{1} \leq t^{-l} \sum_{j=1}^{k}\left(\begin{array}{c}k \\ j\end{array}\right)\left\|\Delta_{t}^{l} g\right\|_{1}\left(2^{k}-1\right) t^{-l} \omega_{1}^{l}(t, g)$.

Hence $N_{i}=2^{k}-1$ for $i=0,1, \ldots, k$, which was to be proved. 
Proof of Lemma 5 . Let $f \in W_{1}^{k}(\mathbb{R})$. Applying Taylor's formula we deduce from (6.5) that at each $x \in \mathbb{R}$,

$$
\begin{aligned}
\pi_{h} f(x)= & \int_{\mathbb{R}} W_{h}(x, z)\left\{\sum_{j=0}^{k} \frac{z^{j} h^{j}}{j !} D^{j} f(x)\right. \\
& \left.\quad+\frac{z^{k} h^{k}}{(k-1) !} \int_{0}^{1}(1-\xi)^{k-1} \Delta_{\xi z h} D^{k} f(x) d \xi\right\} d z .
\end{aligned}
$$

This formula can be transformed to

$$
\begin{aligned}
\pi_{h} f(x)= & \sum_{j=0}^{k} \frac{h^{j}}{j !} \psi_{j h}(x) D^{j} f(x) \\
& +\frac{h^{k}}{(k-1) !} \int_{\mathbb{R}} W_{h}(x, z) z^{k} \int_{0}^{1}(1-\xi)^{k-1} \Delta_{\xi z h} D^{k} f(x) d \xi d z .
\end{aligned}
$$

Applying assumption (6.6) and Hölder's inequality we obtain estimate (6.7).

Proof of Lemma 6 . First, let $f \in W_{1}^{1}(\mathbb{R})$. Introducing a new variable into the second integral, $y=\Delta_{0} \phi_{m}(x-(m-1) h) / 2 h\left(\right.$ where $\left.\Delta_{0} \phi_{m}=\phi_{m+1}-\phi_{m-1}\right)$, we deduce that

$$
\begin{aligned}
\varphi_{m, h}(f) \leq & \int_{(m-1) h}^{(m+1) h}\left|f(x)-f\left(\phi_{m-1}+\frac{\Delta_{0} \phi_{m}}{2 h}(x-(m-1) h)\right)\right| d x \\
& +\left|1-\frac{2 h}{\Delta_{0} \phi_{m}}\right| \int_{\phi_{m-1}}^{\phi_{m+1}}|f(y)| d y .
\end{aligned}
$$

According to our assumptions,

$$
\left|x-\left(\phi_{m-1}+\frac{\Delta_{0} \phi_{m}}{2 h}(x-(m-1) h)\right)\right| \leq h \quad \text { and } \quad\left|1-\frac{2 h}{\Delta_{0} \phi_{m}}\right| \leq B h e^{B h} .
$$

Hence

$$
\varphi_{m, h}(f) \leq \int_{(m-1) h}^{(m+1) h} \int_{x-h}^{x+h}|D f(y)| d y d x+B h e^{B h} \int_{\phi_{m-1}}^{\phi_{m+1}}|f(y)| d y,
$$

and therefore

$$
\sum_{m \in \mathbb{Z}} \varphi_{m, h}(f) \leq 2 B h e^{B h}\|f\|_{1}+4 h\|D f\|_{1} .
$$

At the same time, for any two functions $f, f^{\prime} \in L_{1}(\mathbb{R})$,

$$
\sum_{m \in \mathbb{Z}}\left|\varphi_{m, h}(f)-\varphi_{m, h}\left(f^{\prime}\right)\right| \leq 4\left\|f-f^{\prime}\right\|_{1} .
$$

Using Lemma 4 with $X=l_{1}, \Phi(f)=\left(\varphi_{m, h}(f)\right)_{m \in \mathbb{Z}}$, and remark (6.4), we obtain (6.8). 
Proof of Lemma 7. We use Lemma 4. First, we have

$$
\left\|\Delta_{h}(f(\varphi(\cdot)) D \varphi)\right\|_{1}=\int_{c}^{d-h}|f(\varphi(x+h)) D \varphi(x+h)-f(\varphi(x)) D \varphi(x)| d x .
$$

Let us take $g \in W_{1}^{1}(\mathbb{R})$. Then

$$
\begin{aligned}
\int_{c}^{d-h} \mid g( & (x+h)) D \varphi(x+h)-g(\varphi(x)) D \varphi(x) \mid d x \\
& \leq \int_{c}^{d-h}\left|D \varphi(x+h) \int_{\varphi(x)}^{\varphi(x+h)} D g(y) d y+\Delta_{h} D \varphi(x) g(\varphi(x))\right| d x \\
& \leq \int_{c}^{d-h} D \varphi(x+h) \int_{\varphi(x+h)-P_{1} h}^{\varphi(x+h)}|D g(y)| d y d x+\int_{c}^{d-h}\left|\Delta_{h} D \varphi(x)\right||g(\varphi(x))| d x .
\end{aligned}
$$

Introducing new variables of integration, $z=\varphi(x+h)$ in the first integral, and $z=\varphi(x)$ in the second one, and using (7.2), we obtain

$$
\| \Delta_{h}\left(g(\varphi(\cdot)) D \varphi\left\|_{1} \leq P_{1} h\right\| D g\left\|_{1}+P_{2} \omega_{\infty}^{1}(h, D \varphi)\right\| g \|_{1} .\right.
$$

At the same time, if $f, f^{\prime} \in L_{1}(a, b)$ then

$$
\begin{aligned}
& \left\|\Delta_{h}(f(\varphi(\cdot)) D \varphi)-\Delta_{h}\left(f^{\prime}(\varphi(\cdot)) D \varphi\right)\right\|_{1} \\
& \quad \leq \int_{c}^{d-h} D \varphi(x+h)\left|\left(f-f^{\prime}\right)(\varphi(x+h))\right|+D \varphi(x)\left|\left(f-f^{\prime}\right)(\varphi(x))\right| d x \\
& \quad \leq 2\left\|\mathcal{P} f-\mathcal{P} f^{\prime}\right\|_{1} .
\end{aligned}
$$

Thus, applying Lemma 4 with (6.4) we obtain (6.9).

Proof of (6.10). For almost every $x \in((a+b) / 2, b)$ we have

$$
g(x)=\frac{1}{\delta} \int_{a}^{x}\left(g(y)+\int_{y}^{x} D g(z) d z\right) d y, \quad \text { where } \delta=x-a>\frac{b-a}{2},
$$

and therefore

$$
|g(x)| \leq \frac{1}{\delta} \int_{a}^{x}|g(y)| d y+\frac{1}{\delta} \int_{a}^{x}|D g(z)| \int_{a}^{z} d y d z \leq \frac{2}{b-a}\|g\|_{1}+\|D g\|_{1} .
$$

Similarly, if $x \in(a,(a+b) / 2)$ then $g(x)=(1 /(b-x)) \int_{x}^{b}\left(g(y)-\int_{x}^{y} D g(z) d z\right) d y$. Hence, formula (6.10) is proved.

Proof of (6.11). For almost every $x \in \mathbb{R}$,

$$
g(x)=\int_{-\infty}^{x} D g(y) d y=-\int_{x}^{\infty} D g(y) d y .
$$


Thus,

$$
|g(x)| \leq \min \left(\int_{-\infty}^{x}|D g(y)| d y, \int_{x}^{\infty}|D g(y)| d y\right) \leq \frac{1}{2}\|D g\|_{1},
$$

which was to be proved.

Proof of (1.4). For every $t \in I, \omega_{1}^{k}\left(h, u^{t}\right) \rightarrow 0$ as $h \rightarrow 0$, hence

$$
\forall \delta>0 \forall t>0 \exists \varepsilon=\varepsilon(t) \forall z<\varepsilon \quad\left\|\Delta_{z}^{k} u^{t}\right\|_{1} \leq \frac{\delta}{2} .
$$

At the same time, the function $t \mapsto \mathcal{P} u^{t}$ is continuous on $I$, thus

$$
\forall \delta>0 \exists \eta \forall s, t|s-t| \leq \eta \Rightarrow \forall z\left\|\Delta_{z}^{k}\left(\mathcal{P} u^{s}-\mathcal{P} u^{t}\right)\right\|_{1} \leq \frac{\delta}{2} .
$$

Let us take the numbers $0=t_{0}<t_{1}<\ldots<t_{n}=T$ such that $t_{i+1}-t_{i}<2 \eta$, and $\bar{\varepsilon}=\min \left(\varepsilon\left(t_{0}\right), \ldots, \varepsilon\left(t_{n}\right)\right)$. Then for every $t \in I$, if $t_{i}$ is the point nearest to $t$, and $z<\bar{\varepsilon}$, we have

$$
\left\|\Delta_{z}^{k} u^{t}\right\|_{1} \leq\left\|\Delta_{z}^{k} u^{t_{i}}\right\|_{1}+\left\|\Delta_{z}^{k}\left(\mathcal{P} u^{t}-\mathcal{P} u^{t_{i}}\right)\right\|_{1} \leq \delta
$$

which was to be proved.

\section{Proofs of the main results}

Proof of Theorem 1. First, we show that the functions $a_{\varrho}=a(\cdot, \varrho(\cdot))$ and $a_{\varrho+}^{\prime}, a_{\varrho-}^{\prime}$ defined in (2.12) are continuous on $I$. If $s, t$ are fixed, we have

$$
\begin{aligned}
\left|a_{\varrho}(s)-a_{\varrho}(t)\right| & \leq|a(s, \varrho(s))-a(s, \varrho(t))|+|a(s, \varrho(t))-a(t, \varrho(t))| \\
& \leq \sigma_{30}(|s-t|), \\
\sigma_{30}(\varepsilon) & =A^{\prime} M \varepsilon+\frac{1}{2} \sigma_{10}(\varepsilon) ;
\end{aligned}
$$

the last inequality follows from (2.2), (2.11) and (6.11). Similarly, if $\varrho(s) \leq \varrho(t)$ then applying (2.13), (2.14) and (6.10) we obtain

$$
\begin{aligned}
\left|a_{\varrho+}^{\prime}(s)-a_{\varrho+}^{\prime}(t)\right| \leq & |D a(s, \varrho(s)+0)-D a(s, \varrho(t))| \\
& +|D a(s, \varrho(t))-D a(t, \varrho(t)+0)| \leq \sigma_{40}(|s-t|), \\
\sigma_{40}(\varepsilon)= & A^{\prime \prime} M \varepsilon+\sigma_{20}(\varepsilon) .
\end{aligned}
$$

A similar estimate can be obtained for $a_{\varrho-}^{\prime}$, hence, $a_{\varrho}, a_{\varrho+}^{\prime}, a_{\varrho-}^{\prime}$ are continuous.

Therefore, since (2.10) is assumed, $a_{\varrho}-\varrho^{\prime}$ has a constant sign on $I$. Without loss of generality we may suppose that it is negative, hence

$$
\forall s \in I \quad \beta(s)=\varrho^{\prime}(s)-a(s, \varrho(s)) \geq \beta_{0} .
$$

Let now $s, t$ be fixed, let $\eta=t-s>0$ and

$$
\left[x^{\prime}, x^{\prime \prime}\right]=\{x \in \mathbb{R}: \exists \psi(x) \in[s, t] \lambda(\psi(x), t, x)=\varrho(\psi(x))\} .
$$

Differentiating the definition of $\psi$ we deduce that

$$
D \psi(x)=D \lambda(\psi(x), t, x) / \beta(\psi(x)) .
$$


Hence

$$
1 / C_{1} \leq D \psi(x) \leq C_{2}, \quad C_{1}=\Lambda_{s t}(A+M), \quad C_{2}=\Lambda_{s t} / \beta_{0},
$$

and there exists a function $\xi$ inverse to $\psi$, that is, $\xi(\psi(x))=x$ for $x \in\left[x^{\prime}, x^{\prime \prime}\right]$. For later convenience, we extend $\psi$ onto $\mathbb{R}$, setting $\psi(x)=s$ for $x<x^{\prime}, \psi(x)=t$ for $x>x^{\prime \prime}$. Differentiating (2.4) we obtain

$$
\begin{aligned}
D^{2} \lambda(s, t, x)=-D \lambda(s, t, x) \int_{s}^{t} D^{2} a(\theta, \lambda(\theta, t, x)) D \lambda(\theta, t, x) d \theta & \\
& \text { if } x<x^{\prime} \text { or } x>x^{\prime \prime} .
\end{aligned}
$$

Now, let $x \in \mathbb{R}$ and $h>0$. Let $\nu(\theta)=\lambda(\theta, t, x), \nu_{h}(\theta)=\lambda(\theta, t, x+h)$. It follows from (2.5) that

$$
\nu_{h}(\theta)-\nu(\theta) \leq \Lambda_{s t} h=e^{A^{\prime} \eta} h .
$$

Applying (2.4) and (6.12) we deduce that there exists a number $\xi_{h} \in(0,1)$ such that

$$
\begin{aligned}
D \lambda(s, t, x+h)-D \lambda(s, t, x)= & -\left(\xi_{h} D \lambda(s, t, x)+\left(1-\xi_{h}\right) D \lambda(s, t, x+h)\right) \\
& \times \int_{s}^{t}\left(D a\left(\theta, \nu_{h}(\theta)\right)-D a(\theta, \nu(\theta))\right) d \theta .
\end{aligned}
$$

We divide the interval of integration into three parts: $(s, \psi(x)) \cup(\psi(x), \psi(x+h))$ $\cup(\psi(x+h), t)$, and use assumption $(2.9)$ to the first and third parts:

$$
\begin{aligned}
\int_{s}^{t} & \left(D a\left(\theta, \nu_{h}(\theta)\right)-D a(\theta, \nu(\theta))\right) d \theta=\int_{s}^{\psi(x)} \int_{\nu(\theta)}^{\nu_{h}(\theta)} D^{2} a_{>}(\theta, y) d y d \theta \\
+ & \int_{\psi(x)}^{\psi(x+h)}\left(D a\left(\theta, \nu_{h}(\theta)\right)-D a(\theta, \nu(\theta))\right) d \theta+\int_{\psi(x+h)}^{t} \int_{\nu(\theta)}^{\nu_{h}(\theta)} D^{2} a_{<}(\theta, y) d y d \theta .
\end{aligned}
$$

We deduce from (8.7), (2.5), (8.8), (8.6), (2.13), (8.5) and (2.2) that

$$
|D \lambda(s, t, x+h)-D \lambda(s, t, x)| \leq C_{3} h, \quad C_{3}=\Lambda_{s t}^{2}\left(\eta A^{\prime \prime}+2 A^{\prime} / \beta_{0}\right) .
$$

Hence, $D \lambda(s, t, \cdot)$ is Lipschitz-continuous on $\mathbb{R}$ and therefore $D^{2} \lambda(s, t, \cdot)$ is bounded.

Further, we see that $h^{-1}\left(\nu_{h}(\theta)-\nu(\theta)\right) \rightarrow D \lambda(\theta, t, x)$ and $h^{-1}(\psi(x+h)-$ $\psi(x)) \rightarrow D \psi(x)$ as $h \rightarrow 0$. Next, $\nu(\theta)<\varrho(\theta)<\nu_{h}(\theta)$ if $\theta \in(\psi(x), \psi(x+h))$. Hence, it follows from (8.7) and (8.8) (majorized convergence of integrals) that

$$
\begin{gathered}
D^{2} \lambda(s, t, x)=-D \lambda(s, t, x)\left(B_{>}(x)-B(x)+B_{<}(x)\right), \quad \text { where } \\
B_{>}(x)=\int_{s}^{\psi(x)} b_{>}(\theta, x) d \theta, \quad B_{<}(x)=\int_{\psi(x)}^{t} b_{<}(\theta, x) d \theta \\
B(x)=D \psi(x) a_{\varrho \Delta}^{\prime}(\psi(x)),
\end{gathered}
$$


$b_{\varepsilon}(\theta, x)=D \lambda(\theta, t, x) D^{2} a_{\varepsilon}(\theta, \lambda(\theta, t, x)), \varepsilon$ stands for $<$ or $>, a_{\varrho \Delta}^{\prime}$ is defined in (2.12). Therefore, due to (2.5), (2.13), (8.5), and (2.12), the first inequality in (2.15) is true. Next, changing the order of integration we deduce that

$$
\begin{aligned}
& \left\|B_{>}\right\|_{1} \leq \int_{s}^{t} \int_{\xi(\theta)}^{\infty}\left|b_{>}(\theta, x)\right| d x d \theta=\int_{s}^{t}\left\|D^{2} a_{>}^{\theta}\right\|_{1} d \theta \leq \eta\left\|D^{2} a_{>}\right\|_{*}, \\
& \left\|B_{<}\right\|_{1} \leq \eta\left\|D^{2} a_{<}\right\|_{*} .
\end{aligned}
$$

Setting $\theta=\psi(x)$ and using (2.12) we obtain

$$
\|B\|_{1}=\int_{s}^{t}\left|a_{\varrho \Delta}^{\prime}(\theta)\right| d \theta \leq \eta A_{\Delta}^{\prime} .
$$

Thus, applying (2.5) and (2.13) we prove that the second inequality in (2.15) is true. Now, we want to show (2.16). First, we see from (8.10) that

$$
\begin{gathered}
\left\|\Delta_{h} D^{2} \lambda(s, t, \cdot)\right\|_{1} \leq \omega_{\infty}^{1}(h, D \lambda(s, t, \cdot))\left(\left\|B_{>}\right\|_{1}+\left\|B_{<}\right\|_{1}+\|B\|_{1}\right) \\
+\|D \lambda(s, t, \cdot)\|_{\infty} \omega_{1}^{1}\left(h, B_{>}-B+B_{<}\right) .
\end{gathered}
$$

The first component has just been estimated; let us consider the second. First, according to (8.10),

$$
\left|\Delta_{h} B_{>}(x)\right| \leq \int_{s}^{\psi(x)}\left|\Delta_{h} b_{>}^{\theta}(x)\right| d \theta+\int_{\psi(x)}^{\psi(x+h)}\left|b_{>}(\theta, x+h)\right| d \theta .
$$

The first term on the right-hand side can be estimated by use of Lemma 7 with $f=D^{2} a_{>}^{\theta}, \varphi=\lambda(\theta, t, \cdot)$ :

$$
\begin{aligned}
\int_{\mathbb{R}} \int_{s}^{\psi(x)}\left|\Delta_{h} b_{>}^{\theta}(x)\right| d \theta d x=\int_{s}^{t} \int_{\xi(\theta)}^{\infty}\left|\Delta_{h} b_{>}^{\theta}(x)\right| d x d \theta \\
\leq \int_{s}^{t}\left(\left(3+\Lambda_{s t}\right) \omega_{1}^{1}\left(h, D^{2} a_{>}^{\theta}\right)+\Lambda_{s t} \omega_{\infty}^{1}(h, D \lambda(\theta, t, \cdot)) A_{*}^{\prime \prime}\right) d \theta
\end{aligned}
$$

the second - from (2.5) and (2.13):

$$
\int_{\mathbb{R}} \int_{\psi(x)}^{\psi(x+h)}\left|b_{>}(\theta, x+h)\right| d \theta d x=\int_{s}^{t} \int_{\xi(\theta)}^{\xi(\theta)+h}\left|b_{>}(\theta, x)\right| d x d \theta \leq h \eta \Lambda_{s t} A_{*}^{\prime \prime} .
$$

Combining these two inequalities and applying (8.9) and (2.14) we obtain

$$
\omega_{1}^{1}\left(h, B_{>}\right) \leq \sigma_{3}(h), \quad \sigma_{3}(h)=\eta\left(\left(3+\Lambda_{s t}\right) \sigma_{2}(h)+h \Lambda_{s t} A_{*}^{\prime \prime}\left(1+C_{3}\right)\right) .
$$

The same estimate holds for $B_{<}$. Further, it follows from (8.4) that if $x^{\prime} \leq x \leq$ $x^{\prime \prime}-h$ then

$$
\begin{aligned}
\left|\Delta_{h} D \psi(x)\right| \leq & |D \lambda(\psi(x+h), t, x+h)-D \lambda(\psi(x), t, x)| / \beta_{0} \\
& +\Lambda_{s t}\left|\beta(\psi(x+h))^{-1}-\beta(\psi(x))^{-1}\right| .
\end{aligned}
$$


Applying (2.4), (6.12), (2.5), and next (8.5) and (2.2) we deduce that

$$
\begin{aligned}
\mid D \lambda(\psi(x+h), t, x+h)- & D \lambda(\psi(x), t, x+h) \mid \\
& \leq \Lambda_{s t} \int_{\psi(x)}^{\psi(x+h)}|D a(\theta, \lambda(\theta, t, x+h))| d \theta \leq h \Lambda_{s t}^{2} A^{\prime} / \beta_{0} .
\end{aligned}
$$

Hence, due to (8.9),

$$
|D \lambda(\psi(x+h), t, x+h)-D \lambda(\psi(x), t, x)| \leq C_{4} h, \quad C_{4}=C_{3}+\Lambda_{s t}^{2} A^{\prime} / \beta_{0} .
$$

Further, it follows from the definition (8.3) of $\beta$, and from (2.11), (8.1) and (8.5) that

$$
\left|\beta(\psi(x+h))^{-1}-\beta(\psi(x))^{-1}\right| \leq \beta_{0}^{-2}|\beta(\psi(x+h))-\beta(\psi(x))| \leq \beta_{0}^{-2} \sigma_{4}(h),
$$

where $\sigma_{4}(h)=\sigma_{0}\left(C_{2} h\right)+\sigma_{30}\left(C_{2} h\right)$. Applying these inequalities in (8.15) we conclude that

$$
\omega_{\infty}^{1}(h, D \psi) \leq \sigma_{5}(h), \quad \sigma_{5}(h)=\left(C_{4} h+C_{2} \sigma_{4}(h)\right) / \beta_{0} .
$$

Thus, using Lemma 7 with $\varphi=\psi, f=a_{\varrho \Delta}^{\prime}$ and taking into account (8.5) we obtain

$$
\omega_{1}^{1}(h, B) \leq\left(3+C_{2}\right) \omega_{1}^{1}\left(h, a_{\varrho \Delta}^{\prime}\right)+C_{1} \sigma_{5}(h)\left\|a_{\varrho \Delta}^{\prime}\right\|_{1} .
$$

Finally, due to (8.2) and (2.12), we have

$$
\omega_{1}^{1}(h, B) \leq \sigma_{6}(h), \quad \sigma_{6}(h)=\eta\left(6+2 C_{2}\right) \sigma_{40}(h)+C_{1} A_{\Delta}^{\prime} \sigma_{5}(h) T .
$$

Applying inequalities (8.9), (8.11), (8.12), (2.5), (8.14) and (8.16) to (8.13) we conclude that $(2.16)$ is true and $\sigma(h)=C_{3} h \eta\left(A_{*}^{\prime \prime}+A_{\Delta}^{\prime}\right)+\Lambda_{s t}\left(2 \sigma_{3}(h)+\sigma_{6}(h)\right)$.

Proof of Theorem 2. Step 1. Formula (3.2) yields for each $(n \tau, m h) \in$ $\Omega_{h}^{\prime}$ the equality

$$
z_{m}^{n+1}=\frac{1}{2}\left(1-\mu \alpha_{m+1}^{n}\right) z_{m+1}^{n}+\frac{1}{2}\left(1+\mu \alpha_{m-1}^{n}\right) z_{m-1}^{n}+\tau\left(L_{h} z_{h}\right)_{m}^{n} .
$$

Since (4.3) is satisfied, we have

$$
\mu\|\alpha\|_{\infty} \leq 1
$$

Therefore, the coefficients in (8.17) are nonnegative and $\left\|z^{n+1}\right\|_{1} \leq\left\|z^{n}\right\|_{1}+$ $\tau\left\|\left(L_{h} z_{h}\right)^{n}\right\|_{1}$, and we deduce by induction that

$$
\left\|z^{n}\right\|_{1} \leq\left\|z^{0}\right\|_{1}+\tau \sum_{j=0}^{n-1}\left\|\left(L_{h} z_{h}\right)^{j}\right\|_{1} \quad\left(0 \leq n \leq N_{h}\right) .
$$

But, from (4.2), (4.5) and (3.3), $z^{0}=0$ and $L_{h} z_{h}=L_{h} v_{h}-L_{h} r_{h} u=-L_{h} r_{h} u$. Hence

$$
\left\|z^{n}\right\|_{1} \leq \tau \sum_{j=0}^{n-1}\left\|\left(L_{h} r_{h} u\right)^{j}\right\|_{1}
$$

Thus, we must estimate the norm of $\left(L_{h} r_{h} u\right)^{n}\left(0 \leq n \leq N_{h}-1\right)$. 
Step 2. Let $h \in H$ and $n\left(0 \leq n \leq N_{h}-1\right)$ be fixed and let us introduce the following notation:

$$
\begin{gathered}
f=u^{n \tau}, \quad f^{+}=u^{(n+1) \tau}, \quad \varphi(x)=\lambda(0, n \tau, x), \\
\lambda_{m}=\lambda(n \tau,(n+1) \tau, m h), \quad \phi_{m}(\theta)=\lambda(\theta,(n+1) \tau, m h), \\
\kappa_{m}^{n}=\kappa(n \tau,(n+1) \tau, m h) .
\end{gathered}
$$

It follows from $(2.3),(4.3)$ and $(2.5)$ that for every $m \in \mathbb{Z}$,

$$
\begin{aligned}
\left|m h-\phi_{m}(\theta)\right| & \leq A((n+1) \tau-\theta) \leq A \tau \leq h ; \\
h e^{-A^{\prime} \tau} & \leq \phi_{m+1}(\theta)-\phi_{m}(\theta) \leq h e^{A^{\prime} \tau} .
\end{aligned}
$$

According to (3.2) and (4.1) we have

$\left(L_{h} r_{h} u\right)_{m}^{n}=\frac{1}{2 \tau h}\left[\int_{(m-1) h}^{(m+1) h} f^{+}-\frac{1}{2} \int_{(m-2) h}^{(m+2) h} f\right]+\frac{1}{4 h^{2}}\left[\alpha_{m+1}^{n} \int_{m h}^{(m+2) h} f-\alpha_{m-1}^{n} \int_{(m-2) h}^{m h}\right]$.

Since $u$ is a solution of problem (1.1)-(1.2), it follows from (2.18) that

$$
\int_{(m-1) h}^{(m+1) h} f^{+}=\int_{\lambda_{m-1}}^{\lambda_{m+1}} f
$$

Thus, $\left(L_{h} r_{h} u\right)^{n}$ can be written as $l_{h} f$ where $l_{h}$ is an operator acting from $L_{1}^{\text {loc }}(\mathbb{R})$ to $m\left(\mathbb{R}_{h}\right)$, defined by

$$
\begin{aligned}
\left(l_{h} f\right)_{m}= & \frac{1}{2 \tau h}\left[\int_{\lambda_{m-1}}^{\lambda_{m+1}} f-\frac{1}{2} \int_{(m-2) h}^{(m+2) h} f\right] \\
& +\frac{1}{4 h^{2}}\left[\alpha_{m+1}^{n} \int_{m h}^{(m+2) h} f-\alpha_{m-1}^{n} \int_{(m-2) h}^{m h} f\right] .
\end{aligned}
$$

If we define the prolongation operator (cf. [2]), $p_{h}^{0}: m\left(\mathbb{R}_{h}\right) \rightarrow L_{\infty}^{\text {loc }}(\mathbb{R})$, by

$$
\left(p_{h}^{0} w_{h}\right)(x)=\sum_{m \in \mathbb{Z}} w_{m} \chi_{[0,1)}(x / h-m)
$$

(where $\chi_{A}$ is the characteristic function of $A$ ), then we can see that $\left\|p_{h}^{0} w_{h}\right\|_{1}=$ $\left\|w_{h}\right\|_{1}$ and thus

$$
\left\|\left(L_{h} r_{h} u\right)^{n}\right\|_{1}=\left\|p_{h}^{0} l_{h} f\right\|_{1} .
$$

Step 3. We prove here that the operator $\pi_{h}=p_{h}^{0} l_{h}$ satisfies the assumptions of Lemma 5. First, we show that (6.5) and (6.6) hold. It follows from (8.22), (8.23) and (4.2) that for $m \in \mathbb{Z}, \xi \in[0,1)$,

$$
\begin{aligned}
W_{h}((m+\xi) h, \cdot)= & \frac{1}{2 \tau}\left[\chi_{\left[-1-\mu \kappa_{m-1}^{n}-\xi, 1-\mu \kappa_{m+1}^{n}-\xi\right)}-\frac{1}{2} \chi_{[-2-\xi, 2-\xi)}\right] \\
& +\frac{1}{4 h}\left[\alpha_{m+1}^{n} \chi_{[-\xi, 2-\xi)}-\alpha_{m-1}^{n} \chi_{[-2-\xi,-\xi)}\right] .
\end{aligned}
$$


Thus, (8.18) and (2.6) imply that $\left\|W_{h}\right\|_{\infty} \leq 3 /(4 \tau), \beta_{h}=3$. Next, the following formulas are true for $x=(m+\xi) h, 0 \leq \xi<1$ :

$$
\begin{aligned}
\psi_{0 h}(x)= & \frac{1}{2 h} \Delta_{0}\left(\alpha^{n}-\kappa^{n}\right)_{m}, \\
\psi_{1 h}(x)= & \frac{\mu}{4 h} \Delta_{0} \kappa_{m}^{n}\left(\kappa_{m+1}^{n}+\kappa_{m-1}^{n}\right) \\
& +\frac{1}{2 h}\left[(1-\xi)(\alpha-\kappa)_{m+1}^{n}+(1+\xi)(\alpha-\kappa)_{m-1}^{n}\right],
\end{aligned}
$$

where $\Delta_{0} w_{m}=w_{m+1}-w_{m-1}$. We want to estimate $\left\|\psi_{0 h}\right\|_{1}$ and $\left\|\psi_{1 h}\right\|_{\infty}$. First, by (8.25) we deduce that

$$
\begin{aligned}
\left\|\psi_{0 h}\right\|_{1} & =\frac{1}{2} \sum_{m \in \mathbb{Z}}\left|\Delta_{0}\left(\alpha^{n}-\kappa^{n}\right)_{m}\right| . \\
\left\|\psi_{1 h}\right\|_{\infty} & \leq \frac{\mu}{2 h}\left\|\Delta_{0} \kappa^{n}\right\|_{\infty}\left\|\kappa^{n}\right\|_{\infty}+\frac{1}{h}\left\|\alpha^{n}-\kappa^{n}\right\|_{\infty} .
\end{aligned}
$$

It follows from (4.2), (8.20) and (2.3) that

$$
\begin{aligned}
(\alpha-\kappa)_{m}^{n} & =\frac{1}{\tau} \int_{n \tau}^{(n+1) \tau}\left(a(n \tau, m h)-a\left(\theta, \phi_{m}(\theta)\right)\right) d \theta \\
& =\frac{1}{\tau} \int_{n \tau}^{(n+1) \tau}\left(a(n \tau, m h)-a(\theta, m h)-\int_{m h}^{\phi_{m}(\theta)} D a(\theta, y) d y\right) d \theta
\end{aligned}
$$

Applying estimate (6.11) and using (2.11) we obtain

$$
|a(n \tau, m h)-a(\theta, m h)| \leq\left\|a^{n \tau}-a^{\theta}\right\|_{\infty} \leq \frac{1}{2}\left\|D a^{n \tau}-D a^{\theta}\right\|_{1} \leq \frac{1}{2} \sigma_{10}(\tau) ;
$$

formula (8.21) and assumption (2.2) imply that

$$
\int_{m h}^{\phi_{m}(\theta)}|D a(\theta, y)| d y \leq A A^{\prime}((n+1) \tau-\theta) .
$$

Combining this inequality with (8.29) and (8.28) we obtain

$$
\left|\alpha_{m}^{n}-\kappa_{m}^{n}\right| \leq \frac{1}{2}\left(\sigma_{10}(\tau)+\tau A A^{\prime}\right) .
$$

Next, we estimate $\Delta_{0} \kappa$. By (2.3), (2.2) and (8.21), for fixed $n, m$,

$$
\left|\Delta_{0} \kappa_{m}^{n}\right| \leq \frac{1}{\tau} \int_{n \tau}^{(n+1) \tau} \int_{\phi_{m-1}(\theta)}^{\phi_{m+1}(\theta)}|D a(\theta, y)| d y d \theta \leq 2 h A^{\prime} e^{A^{\prime} \tau}
$$

Finally, let us consider $\Delta_{0}\left(\alpha^{n}-\kappa^{n}\right)_{m}$. It follows from (4.2) and (2.3) that 


$$
\begin{aligned}
\Delta_{0}\left(\alpha^{n}-\kappa^{n}\right)_{m}= & \frac{1}{\tau} \int_{n \tau}^{(n+1) \tau}\left\{\int_{(m-1) h}^{(m+1) h}(D a(n \tau, y)-D a(\theta, y)) d y\right. \\
& \left.+\int_{(m-1) h}^{(m+1) h} D a(\theta, y) d y-\int_{\phi_{m-1}(\theta)}^{\phi_{m+1}(\theta)} D a(\theta, y) d y\right\} d \theta .
\end{aligned}
$$

We first have

$$
\sum_{m \in \mathbb{Z}} \int_{(m-1) h}^{(m+1) h}|D a(n \tau, y)-D a(\theta, y)| d y=2\left\|D a^{n \tau}-D a^{\theta}\right\|_{1} \leq 2 \sigma_{10}(\tau) .
$$

To estimate the remaining part of our sum, let us observe that if we take $f=D a^{\theta}$, $\phi_{m}=\phi_{m}(\theta), B=A^{\prime} \mu$, then the assumptions of Lemma 6 are satisfied due to $(8.21)$. Hence,

$$
\sum_{m \in \mathbb{Z}}\left|\int_{(m-1) h}^{(m+1) h} D a(\theta, y) d y-\int_{\phi_{m-1}(\theta)}^{\phi_{m+1}(\theta)} D a(\theta, y) d y\right| \leq 10 \omega_{1}^{1}\left(h, D a^{\theta}\right)+2 A^{\prime} e^{A^{\prime} \tau} \tau\left\|D a^{\theta}\right\|_{1} .
$$

Combining this estimate with (8.33), (4.6), (2.11), (2.2) and (8.32) we obtain

$$
\sum_{m \in \mathbb{Z}}\left|\Delta_{0}\left(\alpha^{n}-\kappa^{n}\right)_{m}\right| \leq 2 \sigma_{10}(\tau)+10 \sigma_{1}(h)+2 A^{\prime} A_{*}^{\prime} e^{A^{\prime} \tau} \tau
$$

Inequalities (8.30), (8.31), (2.6), (4.3) and (8.34) applied to (8.27) and (8.26) imply the estimates

$$
\begin{array}{rlrl}
\left\|\psi_{0 h}\right\|_{1} & \leq \gamma_{0}(h), & \gamma_{0}(h) & =\sigma_{10}(\mu h)+5 \sigma_{1}(h)+\mu h A^{\prime} A_{*}^{\prime} e^{A^{\prime} \mu h}, \\
\left\|\psi_{1 h}\right\|_{\infty} \leq h^{-1} \gamma_{1}(h), & \gamma_{1}(h) & =\frac{1}{2} \sigma_{10}(\mu h)+h A^{\prime}\left(e^{A^{\prime} \mu h}+\frac{1}{2}\right) .
\end{array}
$$

Hence, Lemma 5 yields the inequality

$$
\left\|p_{h}^{0} l_{h} f\right\|_{1} \leq \gamma_{0}(h)\|f\|_{\infty}+\gamma_{1}(h)\|D f\|_{1}+\frac{81}{4 \mu} \omega_{1}^{1}(h, D f) .
$$

S tep 4. We now estimate the terms occurring on the right-hand side of (8.35) by the given numbers. First, it follows from (2.7) that

$$
\begin{gathered}
f(x)=D \varphi(x) \\
\Delta_{h} D f(x)=\Delta_{h} D^{2} \varphi(x) g(\varphi(x+h))+D^{2} \varphi(x) \Delta_{h}(g \circ \varphi)(x) \\
+\Delta_{h}\left((D \varphi)^{2}\right)(x) D g(\varphi(x+h))+(D \varphi(x))^{2} \Delta_{h}(D g \circ \varphi)(x) .
\end{gathered}
$$

Assumption (4.6) implies the estimates

$$
\|D \varphi\|_{\infty} \leq \Lambda, \quad \varphi(x+h)-\varphi(x) \leq \Lambda h, \quad\left\|D^{2} \varphi\right\|_{1} \leq \Lambda^{\prime} .
$$

Hence

$$
\left|\Delta_{h}(g \circ \varphi)(x)\right| \leq \int_{\varphi(x)}^{\varphi(x)+\Lambda h}|D g(y)| d y \leq \Lambda h\|D g\|_{\infty}
$$




$$
\begin{aligned}
&\left|\Delta_{h}\left((D \varphi)^{2}\right)(x)\right| \leq|D \varphi(x+h)+D \varphi(x)| \int_{x}^{x+h}\left|D^{2} \varphi(y)\right| d y \Rightarrow\left\|\Delta_{h}\left((D \varphi)^{2}\right)\right\|_{1} \leq 2 \Lambda \Lambda^{\prime} h ; \\
&\left|\Delta_{h}(D g \circ \varphi)(x)\right| \leq \int_{\varphi(x)}^{\varphi(x)+\Lambda h}\left|D^{2} g(y)\right| d y \quad \\
& \Rightarrow \int_{\mathbb{R}}(D \varphi(x))^{2}\left|\Delta_{h}(D g \circ \varphi)(x)\right| d x \leq \Lambda^{2} h\left\|D^{2} g\right\|_{1} .
\end{aligned}
$$

Consequently,

$$
\begin{gathered}
\|f\|_{\infty} \leq \Lambda\|g\|_{\infty}, \quad\|D f\|_{1} \leq \Lambda^{\prime}\|g\|_{\infty}+\Lambda\|D g\|_{1}, \\
\omega_{1}^{1}(h, D f) \leq \sigma(h)\|g\|_{\infty}+3 \Lambda^{\prime} \Lambda h\|D g\|_{\infty}+\Lambda^{2} h\left\|D^{2} g\right\|_{1} .
\end{gathered}
$$

Combining these inequalities with (8.35), (8.24) and (8.19) and using (6.11), we obtain

$$
\begin{aligned}
\left\|z_{h}\right\|_{*} \leq T\left\{\left[\frac{\Lambda}{2} \gamma_{0}(h)+\left(\Lambda+\frac{\Lambda^{\prime}}{2}\right) \gamma_{1}(h)+\frac{\mu_{1}}{2}\right.\right. & \sigma(h)]\|D g\|_{1} \\
& \left.+h \Lambda \mu_{1}\left(\frac{3}{2} \Lambda^{\prime}+\Lambda\right)\left\|D^{2} g\right\|_{1}\right\} .
\end{aligned}
$$

This estimate implies (4.7), hence the proof of Theorem 2 is complete.

Proof of Theorem 3. The proof is carried out with the use of Lemma 4. Let us consider the Banach space $L\left(\Omega_{h}\right)$ consisting of all mesh functions $y_{h}$ which are bounded and such that the norm $\left\|y_{h}\right\|_{*}$ defined by (3.1) is finite. For $h \in$ $(0,1)$ let the operator $\Phi_{h}: L_{\infty}(\mathbb{R}) \cap L_{1}(\mathbb{R}) \rightarrow L\left(\Omega_{h}\right)$ be defined by $\Phi_{h}(g)=$ $F_{h} g-r_{h}(E(0, \cdot) g)$. We must show that $\Phi_{h}$ satisfies the assumptions of Lemma 4 . First, according to (3.4), $\left\|F_{h} g\right\|_{*} \leq\left\|\left(F_{h} g\right)^{0}\right\|_{1}=\|g\|_{1}$. Next, it follows from formula (2.8) that for every $n>0,\left\|r_{h}^{0}(E(0, n \tau) g)\right\|_{1}=\|E(0, n \tau) g\|_{1}=\|g\|_{1}$. Since $\Phi_{h}$ is linear, we deduce that (6.1) is satisfied for each $h \in H$ and $M=2$.

Next, by Theorem 2 , if $f \in W_{2}^{1}(\mathbb{R})$ then $\left\|z_{h}\right\|_{*} \leq M_{1}(h)\|D f\|_{1}+M_{2} h\left\|D^{2} f\right\|_{1}$. Applying Lemma 4 with $\eta=\max \left(h, M_{1}(h)^{2}\right)$, we obtain inequality (4.8) and the proof is complete.

\section{References}

[1] J. K. Kowalski, Application of box splines to the approximation of Sobolev spaces, J. Approx. Theory 61 (1990), 53-73.

[2] —, A method of approximation of Besov spaces, Studia Math. 96 (1990), 173-183. 